\title{
GRENZBEGEHUNGEN.
}

\section{Eine religionspädagogische Reflexion über die Dimension von Grenzen und Grenzverlusten in der Lebenswelt Jugendlicher und die Suche nach theologischen Anknüpfungspunkten}

David Novakovits
UDK $37: 2$

$316.728-053.6$

128-024.536:398.42

\section{Theologie bzw. Religionspädagogik als Grenz-Gängerin}

Der Kirche als Ekklesia ist schon von Grund auf etwas Grenzüberschreitendes in ihre Definition eingeschrieben: Als Herausgerufene (ek-kalein) hat die kirchliche Gemeinschaft den Auftrag, ihre ureigene Aufgabe nicht in einem abgegrenzten Raum, einen festgelegten Horizont zu suchen, sondern sich vor die Welt gerufen zu fühlen. Man kann das 2. Vatikanische Konzil hier als eine Maßgabe für die Religionspädagogik begreifen, indem erst dort, wo die Grenze nicht mehr als Trennungsscheide von Eigenem und Fremden, von Wahrem und Zufälligem begriffen wird, sich auch in der Religionspädagogik ein neues Paradigma ausbilden konnte, das bis heute zahlreiche Anpassungen erfahren hat, aber im Grunde noch auf dieser veränderten Wahrnehmung des Grenz-Begriffes fußt.

Das 2. Vatikanische Konzil hat die Grenzen, wie sich Kirche in der Welt von heute versteht, überdacht und neu gezogen, und zwar dermaßen, dass die »Freude und Angst, Trauer und Hoffnung der Menschen von heute« (GS 1) nicht mehr das jenseitig Fremde sind, sondern gleichzeitig auch »Freude und Hoffnung, Trauer und Angst der Jünger Christi « ${ }^{1}$. Hier wurde, so könnte man sagen, eine Grenze überschritten. Diese Überschreitung hat auch eine Verbindung geschaffen: Zwischen der Lebenswelt der Menschen heute, insbesondere auch der Kinder und Jugendlichen, und dem theologischen Denken. Genau diese Grenzüberschreitung stellt den fruchtbaren Boden der Religionspädagogik dar, indem hier der Austausch zwischen fachlicher

\footnotetext{
* $\quad$ David Novakovits, Institut für Praktische Theologie, Fachbereich Religionspädagogik und Katechetik, Katholisch-Theologische Fakultät Wien, david.novakovits@univie.ac.at

1 Die pastorale Konstitution über die Kirche in der Welt von heute »Gaudium et spes«, zitiert nach: Karl Rahner, Herbert Vorgrimler, Kleines Konzilskompendium, Freiburg im Breisgau ${ }^{35} 2008,449$.
} 
Theologie und jugendlicher Lebenswelt vorangetrieben wird. Wenn man also nach dem Ort der Religionspädagogik fragen möchte, dann zeigt sie sich als eine Figur an der Schwelle, als eine Grenzgängerin auf der Schwelle zwischen Theologie einerseits und der Lebenswelt von Kindern und Jugendlichen andererseits.

Sich auf einer Schwelle zu bewegen hat auch etwas von einem Drahtseilakt an sich: Die Religionspädagogik versucht, gleichsam balancierend Theologie und die Lebenswelt Jugendlicher miteinander zu verbinden, ohne vom Seil runterzufallen, sprich: Ohne beide Pole unkritisch und undifferenziert zu vermengen. Was in dieser Beschreibung Kontur gewinnt, bezeichnet die Religionspädagogik Korrelation und kann wohl als das religionspädagogische Paradigma schlechthin bezeichnet werden, das sich in der geistigen Atmosphäre des 2. Vatikanums entwickelt hat. Die Balance zu halten, heißt, immer wieder Übersetzungsarbeit zu leisten (und zwar in beiderseitige Richtung!) und so erst ein spannungsgeladenes Gespräch zu eröffnen.

Mittlerweile hat sich das eben formulierte Paradigma in vielfache Richtungen weiterentwickelt. Eine relativ jüngere Ausgestaltung davon ist etwa der Ansatz des Philosophierens und Theologisierens mit Kindern und Jugendlichen, auf den im vierten Teil des Artikels noch Bezug genommen werden wird.

\section{Der Verlust an Grenzen in der Lebenswelt Jugendlicher und der damit einhergehende Verlust von Orientierungsmöglichkeiten}

Wenn man einen Blick in die vielgestaltige Lebenswelt Jugendlicher wirft, kann man Phänomene ausmachen, die mit dem Thema des Forums transgressions - Grenzüberschreitungen in einer engen Beziehung stehen.

Grenz-Erfahrungen gehören traditionell zum Repertoire einer jugendlichen Erfahrung. Dieses (auch provokative) Verhalten des Grenz-Überschreitens wird oft negativ gesehen, aber es beinhaltet auch starke erfahrungserweiternde Dimensionen: Sei es etwa eine mögliche Definition von Lernen als eines ständigen geistigen Überschreitens der Begrenzungen des eigenen Wissenshorizontes oder die fundamentalste Grenzüberschreitung in der Jugendzeit schlechthin: vom Kind zum Erwachsenen, die Loslösung vom elterlichen Horizont und die rebellierende oder auch sanfte Überschreitung desselben.

Grenzen erfüllen wichtige Funktionen: Massimo Recalcati, der gegenwärtig wohl bekannteste italienische Psychoanalytiker, hebt vor allem die formierende Funktion von Grenzen im Subjektivierungsprozess hervor: Er sieht in Grenzen, welche dem jugendlichen Subjekt in seinem individuellen Wachsen gesetzt werden, einen wichtigen Ermöglichungsgrund, den er mit der Bedeutung von Regeln in einem Spiel vergleicht: »Die Erfahrung des 
Spiels ist dort nicht möglich, wo keine Grenzen existieren: Es braucht Grenzen und Schwellen. $\aleph^{2}$ Grenzen, die gesetzt werden, können dem jugendlichen Subjekt als Orientierungspunkte dienen und zu kreativ-schöpferischer Auseinandersetzung provozieren und herausfordern.

Ein Übermaß an Begrenzungen kann zwar mit unterdrückenden Zügen einhergehen, aber viel schwerwiegender als diese repressive Dimension ist für Recalcati gegenwärtig der Mangel an Grenzen bzw. Grenz-Erfahrungen: Eine Vielzahl an Milieus der heutigen Kinder und Jugendlichen wachsen in einem sozialen Umfeld auf, das vielmehr von einem (kapitalistisch geprägten) Diskurs begleitet wird, welcher von der Überflüssigkeit von Grenzen ausgeht. Wie wir wissen, ist dieser Diskurs sehr wirkmächtig. In Bezug auf den Begriff der Grenze könnte man ihn wohl stichwortartig so formulieren: Alles (d.h. alles Waren-förmige) soll jederzeit, ohne reglementierende Begrenzung, verfügbar sein (für alle, die es sich leisten können). Dieser Diskurs stellt die heimtückische Frage, warum wir so etwas wie Grenzen und Begrenzungen in unserem individuellen und gesellschaftlichen Leben überhaupt noch akzeptieren sollten. Indem er die Grenze als Begrenzung der individuellen Freiheit vermittelt (du darfst, kannst, sollst dieses und jenes nicht), kann sich dieser Diskurs im Anschluss als Befreier von diesen limitierenden Fesseln darstellen, indem er unbegrenzte Möglichkeiten verspricht und zu einer Lebensweise aufruft, die vor allem durch das Genießen jenseits aller Einschränkungen geprägt ist. Der kapitalistische Diskurs spricht hier, wenn wir genau hinhören, aber nicht von einer Überschreitung der Grenze, sondern im Grunde von deren Abschaffung: es ist das un-limitierte Vergnügen, das hier schon als beinahe religiöses Versprechen angepriesen wird.

\section{Existenziell-indifferente Jugendliche und die literarische Figur des Zombies}

Diese un-begrenzte gesellschaftliche Situation, die uns umgibt, wirkt aber zutiefst verunsichernd für junge Menschen (so weist etwa auch Beate Großegger, Mitarbeiterin am Institut für Jugendforschung in Wien, darauf hin, dass 7 von 10 jungen Österreicherinnen zwischen 16 und 29 ihre Generation als eine verunsicherte Generation bezeichnen). Dieses Gefühl, dass zwar alles möglich ist, aber wenige gesellschaftliche Orientierungspunkte vorhanden sind, lässt bei Jugendlichen einen Rückzug in eine private, scheinbar heile Welt suchen. In dieser privaten Welt werden aber »modische Lifestyletrends und gesellschaftliche Leitwerte kaum hinterfragt, sondern als Soll-

2 Massimo Recalcati, Il disagio della giovinezza, in: Massimo Recalcati, Elogio del fallimento, Trento 2011, 117-137, 119. (Übersetzung David Novakovits) 
werte weitgehend akzeptiert und im Alltag meist unkritisch angewendet. $\aleph^{3}$. Großegger nennt diesen Typus der Jugendlichen »Zeitgeistsurfer« und rechnet $42 \%$ der jungen ÖsterreicherInnen diesem Segment zu.

Ein individualistischer Kult des Glücks ist deshalb gesellschaftlich hochriskant, weil er keine (Lebens-)Orientierung anbietet, die über eine sofortige Wunscherfüllung von Bedürfnissen hinausgeht. Man könnte diese kurze und sehr grobe Diagnose so schließen: Eine Vielzahl an Milieus der postmodernen Jugend sind gegenwärtig einem hohen Risiko eines Orientierungsverlustes ausgesetzt, was implizit mit dem Wegfall an Grenz-Erfahrungen verbunden ist.

Dies hat nicht nur individuelle, sondern auch enorme gesellschaftliche Konsequenzen. Großegger verweist auf eine Entwicklung bei Jugendlichen, die sie existenziell indifferent werden lässt. Die Bedeutung von »existenziell indifferent « erschließt sich im nachfolgenden Zitat. Die Jugendforscherin hebt hervor, dass dieses spezifische Milieu eng mit dem Phänomen des Orientierungsverlustes verknüpft ist, was nicht zuletzt auch der Theologie zu denken geben muss:

»Was (im Leben dieser Jugendlichen, Anm.) zählt, ist Ablenkung. Nicht selten zeigt sich auch eine Grundhaltung, die die Psychologie als existenzielle Indifferenz beschreibt. Existenziell Indifferente sehen im eigenen Leben zwar keinen Sinn, sie erleben aber auch keine belastende Sinnkrise, sondern kompensieren Sinndefizite: beispielsweise mit freizeitweltlichem Hedonismus und Orientierung an kleinen, schnellen Glücksmomenten. Existenziell Indifferente sind generell (eher) jung, wissens- und technikorientiert, sie haben nicht den Anspruch, etwas von bleibendem Wert zu tun, sind daher für ehrenamtliche Tätigkeiten kaum zu gewinnen, und zeigen insgesamt wenig Leidenschaft und Engagement - egal, ob es um sie selbst oder andere geht. Oberflächlich betrachtet, sind existenziell indifferente Jugendliche zufrieden. Vermutlich deshalb kommen sie in der niemals verstummenden Problemjugenddebatte auch so gut wie nicht vor. Sie machen im Hier und Jetzt eben keine Probleme. Das mag für den Moment vielleicht beruhigen. Es sollte aber nicht darüber hinwegtäuschen, dass existenziell Indifferente ganz sicher nicht diejenigen sind, auf denen ein tragfähiges Gemeinwesen aufbauen kann. $\ll^{4}$

Wenn die In-Differenz, also das Wegfallen irgendeines signifikanten Unterschiedes bis in die innerste Existenz hineinreicht, dann wird es nicht mehr möglich, Bedeutsames von Unbedeutsamen zu scheiden, dann gibt es nichts mehr, »was mich unbedingt angeht« (Tillich).

Spuren dieser existenziell-indifferenten Jugendlichen sind heute in literarischen Zeugnissen klar sichtbar. In der Jugendliteratur hat gegenwärtig die Figur des Zombies eine besondere Bedeutung inne und vielleicht kann

3 Beate Großegger, Zwischen Freakout und Normcore, Jugend und Jugendkulturen in den späten 2010er Jahren, in ÖRF 25 (2017) 1, 7-16, 12.

4 Großegger, Zwischen Freakout und Normcore, 14. 
gerade die Zombie-Figur als ein prägnantes Spiegelbild dieser existenziellen In-Differenz gelesen werden. Man wird das Auftauchen und gegenwärtige Herumtaumeln der Zombie-Figur in zahlreichen Romanen der Jugendliteratur wohl kaum als Zufall bezeichnen können, sondern vielmehr als kulturellen und literarischen Ausdruck einer jugendlichen Generation lesen müssen. Der Zombie ist wohl die existenziell-indifferente Erscheinungsform par excellence.

Was charakterisiert diese Figur des Zombies eigentlich? Man wird schwer behaupten können, dass man mit einem Zombie gut ins Gespräch kommen kann, mit ihm ist keinerlei gefühlsmäßige, affektive Gemeinschaft möglich. Er besteht nur aus dem Körper, und hat keinerlei Orientierung und Reflexionsfähigkeit mehr (man könnte darüber diskutieren, ob das Zutaumeln auf noch lebende Menschen als sinnvolle Orientierungsmöglichkeit angesehen werden kann).

In einem der bekanntesten Romane dieses Genres, Warm Bodies ${ }^{5}$ von Isaac Marion, steht ein noch jugendlicher Zombie, genannt R, im Mittelpunkt. Warm Bodies erzählt die Geschichte aus der Erzähl-Perspektive des Zombie-Jugendlichen R, d.h. man kann in das Innenleben dieses Zombies und seine ungeheure Gedanken und Fragen eintauchen. Der Frage, die nun in den Fokus rücken soll, ist folgende: Wenn wir die Äußerungen des Zombies R nicht nur als ein beliebiges Sinnieren über die Welt wahrnehmen, sondern gleichsam als literarische Verdichtung der geistigen Atmosphäre existenziell indifferenter Jugendlicher sehen, welche Wahrnehmungen können wir dann machen?

Rudolf Englert weist darauf hin, dass die Religionspädagogik »(..) zunächst einmal [fragen muss] (...), wo und wie Religion in der Welt heutiger Kinder und Jugendlicher vorkommt« und darüber hinaus nach den »verschiedenen Formen verborgener Religion $\aleph^{6}$ in der Lebenswelt Jugendlicher zu suchen hat. Kann man, so möchte ich diesen Gedanken aufnehmen, in den literarischen Äußerungen dieses Zombie-Jugendlichen auch eine theologische Dimension, ein theologisches Denken erkennen? An den ersten Teil anschließend: Gibt es auch so etwas wie ein Philosophieren und Theologisieren mit Zombies? Dies soll im Anschluss in bruchstückhafter Weise versucht werden, indem einige Passagen dieses Buches vorgestellt und kommentiert werden, um abschließend Rückschlüsse daraus für die Theologie zu ziehen.

Schon im ersten Satz des Romans drückt sich die grenzen-lose Dimension der Zombie-Existenz aus, indem der Zombie darauf verweist, dass sie

$5 \quad$ Isaac Marion, Warm Bodies, New York 2009.

6 Rudolf Englert, Religionspädagogische Grundfragen. Anstöße zur Urteilsbildung, Stuttgart 2007, 239. 
selbst jene Figuren sind, die noch die letzte, ultimative Grenze, nämlich jene des Todes, überlebt haben. Insofern ist der Zombie nicht einfach tot, sondern kann als eine Existenzform angesehen werden, in der die humane letzte Grenze nicht mehr gilt: »Ich bin tot, aber es ist nicht so schlimm. Ich habe gelernt, damit zu leben. $\ll^{7}$

Wie sieht dieses neue Leben aus? Die Zombies taumeln in diesem Roman durch eine zerstörte Welt, in der letzte Enklaven noch von lebendigen Menschen bevölkert werden. Außerhalb dieser abgeschlossenen, autarken Stätten, nur wenigen zugänglich, läuft der Rest der Menschheit herum. Einer Sprachfähigkeit und eines sinn-vollen Antriebs beraubt, sind sie dennoch nicht jenseits jeder Vernunft. Sie sind einfach aus einer Welt herausgefallen, in der man keine Rolle mehr zu haben scheint, die man einnehmen und ausfüllen könnte:

»Keiner, den ich kenne, hat konkrete Erinnerungen. Bloß die vage, verkümmerte Ahnung einer lange vergangenen Welt. (...) Wir erkennen die Zivilisation wieder (...) aber wir spielen keine Rolle darin. Wir haben keine Geschichte. Wir sind bloß da. Wir tun, was wir tun, die Zeit vergeht, keiner stellt Fragen. Aber wie gesagt: es ist nicht so schlimm. Wir scheinen ohne Verstand, aber wir sind es nicht. Die rostigen Rädchen der Vernunft drehen sich noch (...) Wir grunzen und seufzen, nicken und zucken die Achseln, und manchmal rutschen uns ein paar Worte heraus. Es ist nicht so viel anders als vorher. $\ll^{8}$

Es ist nicht der äußerliche Anschein, das Aussehen, das Lebende und Zombies unterscheidet. Vielmehr ist es das Gefühlsleben und die Lebenseinstellung, die divergieren. Das Fressen ist der einzige Antrieb, der von den zombiehaften Figuren aber nicht gesteuert oder beherrscht wird. Sie sind diesem diffusen Trieb ausgeliefert. Man könnte sagen: Hier erscheint der Zombie wie der perfekte Konsument, da er keine Fragen und Ansprüche hat, sondern den Konsum (man könnte sagen: der Konsum, unter dem andere leiden müssen) zur Existenzform erhoben hat.

Am wirkgewaltigsten ist aber die enorme Sprachlosigkeit, von der das Zusammenleben der Zombies in diesem Roman gezeichnet ist. Hier dazu die Überlegungen von R:

»Ich überlege, was ich sagen könnte, aber mir fällt nichts ein, und wenn mir etwas einfiele, könnte ich es wahrscheinlich nicht sagen. Das ist mein großes Hindernis, der gewaltigste aller Felsbrocken auf meinem Weg. In meiner Vorstellung bin ich wortgewandt; über verschachtelte Wortgerüste erreiche ich die höchsten Decken der Kathedrale, an die ich meine Gedanken male. Mache ich aber den Mund auf, stürzt alles in sich zusammen. (...) Ich weiß nicht, warum wir nicht reden. Ich kann die drückende Stille, die auf unserer Welt liegt, nicht

7 Marion, Warm Bodies, 13.

8 Ebd., 14. 
erklären. (...) Eines der vielen Symptome des Todes? Oder haben wir einfach nichts mehr zu sagen? « ${ }^{9}$

\section{Theologische Anknüpfungspunkte: Die Kraft der Sprache und des Gespräches}

Der Roman stellt die Zombie-Existenz als eine traurige Existenz dar: Ohne die Fähigkeit des sich-Erinnerns, ohne die Erfahrung von Beziehungen zu anderen Menschen (jenseits eines fressen-und-gefressen-werdens). Es zeigt sich hier ein Subjekt, das in der Gefährdung steht, seine Sprachfähigkeit zu verlieren und das sich selbst nicht mehr in der Geschichte verorten kann, ein Subjekt, dass nicht mehr weiß, wie es mit seinen Affekten, Antrieben und Wünschen umgehen soll, und woraufhin es sich orientieren kann.

Meines Erachtens konnten hier drei theologisch bedeutsame Spuren offengelegt werden. Eine Welt jenseits jeglicher Begrenzung führt nicht zu der oft verheißenen letzten Freiheit der Subjekte, sondern zu Welt, die in der Gefahr steht, das zutiefst Humane zu verlieren: Sprache, Geschichtlichkeit, affektive Beziehungsfähigkeit.

Welches Potential kommt dem Religionsunterricht in dieser Situation zu? Vielleicht muss zuerst gesagt werden, worum es keinesfalls gehen kann: Es kann nicht darum gehen, von neuem eine übergeschichtliche und letztgültige, metaphysisch verankerte Ordnung zu propagieren und religiös-autoritäre Grenz-Wächterin des (moralischen) Einhaltens dieser Ordnung zu sein. Hier bewegen wir uns im Wirkungsfeld der Fundamentalisten.

Ein enormes Potential des RU liegt dem gegenüber aber im Horizont des Sprechens selbst. Nicht zuletzt der Zombie R sieht in der Sprache noch etwas verborgen Religiöses, wenn er mit ihr »die höchsten Decken der Kathedrale« erreicht, die sich in seinen Sprach-Gedanken schemenhaft bildet. Die Religionspädagogik (und somit auch die Theologie) muss die Sprache der SchülerInnen als lebenswichtig und todernst nehmen, insofern in der Sprache sich uns der fundamentale Weltzugang eines Subjektes offenbart.

Der Religionsunterricht kann zu diesen Sprechakten motivieren und gleichzeitig die theologischen Spuren in diesem Sprechen wahrzunehmen und als schöpferische Kräfte aufzugreifen versuchen. Hierfür steht etwa der didaktisch relativ neuere Ansatz des Philosophierens und Theologisierens mit Kinder und Jugendlichen, auf den eingangs hingewiesen wurde. Dieser Ansatz versucht, die Sprache der SchülerInnen, ihre Äußerungen, ihre Fragen, Antworten und Argumente wahr-zu-nehmen und auch auf ihre existentiellen, philosophischen und theologischen Implikationen hin 
zu analysieren. In der Verlangsamung und der hohen Aufmerksamkeit für Sprechakte, üben Jugendliche — so das Ziel — das immer genauere Aufeinander-Hören, das Miteinander-Streiten, das kritische Rückfragen und das Aushalten solcher Anfragen an das eigene Sprechen. Sprachphilosophisch, bzw. von Wittgenstein her, wissen wir: Die Grenzen unserer Sprache sind die Grenzen unserer Welt. Die Aufgabe des Religionsunterrichtes könnte darin bestehen, die Sprachwelt der Jugendlichen zu erweitern und zu produktiven, d.h. zu respektvollen, Grenzüberschreitungen zu ermuntern: Aus dem Verstummen in die Sprache, aus der privaten Welt zur Auseinandersetzung mit einander, aus der bloßen »individuellen Meinung« (i.S. von: jeder kann denken, was er will) zur Frage nach Begründungen eigener Weltsicht und eigenen Handelns.

Dieses Einüben des Sprechens geschieht im RU aber nicht auf »neutralem« Boden, sondern auf Grundlage der biblischen Narrative. Die Erzählungen unserer Tradition, so Rudolf Englert, können jene Grammatik bilden, mit Hilfe derer die SchülerInnen ihr sprachliches Bewohnen der Welt einüben können. Dabei kommt den biblischen Texten nicht eine letztgültige Deutungshoheit des Lebens im Sinne einer mathematischen Ableitung zu. Religionspädagogisch essentiell ist es, die Texte nicht als übergeschichtliche Instruktionen zur Lebensführung zu präsentieren (dann wäre die Bibel so etwas wie eine esoterische Lebenshilfe), sondern als ein sprachliches Gegenüber, das die Jugendlichen in eine schöpferische Auseinandersetzung mit diesem Gesprächspartner bringt. Erst so kommt ein Gespräch zustande. Für die Theologie bedeutet dies auch, auf die Fragen und Antworten der Jugendlichen zu hören und diese auf gleicher Augenhöhe anzuerkennen. Vielleicht sprechen wir hier von einer weiteren Grenzüberschreitung, wo »der Hörer/ die Hörerin des Wortes« sich zu einem Gesprächspartner hin transformiert. Das heißt, dass auch die Theologie in diesem Gespräch von den Äußerungen der Jugendlichen lernen kann.

»Die Botschaft ist der Interpretationsmacht anderer Agenten ausgesetzt - wo wird dies intensiver erfahrbar als im Religionsunterricht? Gemäß Gaudium et spes 44 eignet den eigenwilligen Dekonstruktionen, Adaptionen und Neukombinationen von Schülerinnen und Schülern eine kreative Kraft, durch die der Offenbarung neue Sprachpotenziale und Artikulationsgestalten zuwachsen können - wenn sie wiederum von einer Theologie rezipiert werden, die sich (...) offenhält für fremdprophetische Rede, ja für die intellektuelle Schubkraft häretischer Geltungsansprüche. $\ll^{10}$

Der Religionsunterricht hat großes Potential, zu dieser Sprachfähigkeit einen Teil beizutragen. Gerade darin liegt auch eine Brücke verborgen, die

10 Matthias Sellmann, »Ohne pics glaub ich nix!«, Die Jüngeren als Produzenten religiöser Bedeutungen, in: Norbert Mette (Hrsg.), Religionsunterricht als Ort der Theologie, Freiburg 2012, 65-92, 81. 
dem RU ein Gespräch mit anderen Schulfächern leichter und intensiver ermöglichen kann und eine verstärkte Integration in das Schulleben eröffnet: Im Dialog mit künstlerisch und sprachlich orientierten Fächern kann versucht werden, auch gemeinsam an der plural orientierten Entwicklung von Ausdruck, Interpretations- und Sprachfähigkeit der SchülerInnen zu arbeiten. Die eingangs erwähnte Übersetzungsarbeit kann auch bedeuten, über die Grenzen der Schulfächer hinweg Impulse aus dem spezifischen Feld der »biblischen Grammatik« in das säkulare Feld des Schullebens einzubringen und sich so zu einem anfragbaren und vernehmbaren Gesprächspartner zu machen.

Gerade dieses Potential, die Sprachfähigkeit der Kinder und Jugendlichen zu erweitern, kann eine Hilfestellung bieten, jene existenzielle Indifferenz zu überwinden, vor welcher gerade auch das letzte Buch des biblischen Kanons warnt: »Ach, wärst du doch kalt oder heiß!« (Offb 3,15).

\section{Literaturverzeichnis:}

Rudolf Englert, Religionspädagogische Grundfragen. Anstöße zur Urteilsbildung, Stuttgart 2007.

Die pastorale Konstitution über die Kirche in der Welt von heute »Gaudium et spes«, zitiert nach: Karl Rahner, Herbert Vorgrimler, Kleines Konzilskompendium, Freiburg im Breisgau 352008.

Beate Großegger, Zwischen Freakout und Normcore, Jugend und Jugendkulturen in den späten 2010er Jahren, in ÖRF 25 (2017) 1, 7-16.

Isaac Marion, Warm Bodies, New York 2009.

Massimo Recalcati, Il disagio della giovinezza, in: Massimo Recalcati, Elogio del fallimento, Trento 2011, 117-137.

Matthias Sellmann, »Ohne pics glaub ich nix!«, Die Jüngeren als Produzenten religiöser Bedeutungen, in: Norbert Mette (Hrsg. u.a.), Religionsunterricht als Ort der Theologie, Freiburg 2012, 65-92. 
UN-LIMITED LIFE.

\section{A theological perspective for religious education on the contemporary life of youth.}

Religious education is situated on the threshold between the environment of students and theology. As a commuter across these borders religious education knows about the dialectical relation and importance of transgression and protection of borders. The article focuses on the risks of un-limited life, which can be related with a lack of capacity of orientation like the sociological phenomenon of the so-called »existentially-indifferent youth" is showing. The second part tries to connect these analysis with the literary phenomenon of the zombie, as the particular figure of transgressing the ultimate border (the death) and still living - without borders. In the third part the article asks for the theological points of contact for religious education and tries to explain the potential of religious education in the context of a youth which is struggling with necessary transgression of borders and the living in a situation, where borders are more and more eliminated.

KeY worDs: Religious Pedagogics, adolescent live style, zombie, philosophize and theologize with children and adolescents. 